\title{
A population-based study of gefitinib in patients with postoperative recurrent non-small cell lung cancer
}

\author{
KINYA FURUKAWA $^{1}$, JUNZO ISHIDA ${ }^{1}$, MASAHARU INAGAKI $^{2}$, KAZUHIKO TAKABE $^{2}$, \\ SHIGEMI ISHIKAWA ${ }^{3}$, MITSUAKI SAKAI ${ }^{3}$, HIDEO ICHIMURA ${ }^{4}$, KOICHI KAMIYAMA $^{5}$, \\ TAKAYUKI KABURAGI ${ }^{6}$, KENJI HAYASHIHARA ${ }^{7}$, KOJI KISHI $^{1}$, MAKOTO SAITO $^{1}$ and HIROAKI SATOH ${ }^{8}$ \\ ${ }^{1}$ Tokyo Medical University, Ibaraki Medical Center; ${ }^{2}$ Tsuchiura Kyodo General Hospital; \\ ${ }^{3}$ University of Tsukuba Hospital; ${ }^{4}$ Tsukuba Medical Center Hospital; ${ }^{5}$ Tsukuba Kinen Hospital; \\ ${ }^{6}$ Ibaraki Prefectural Central Hospital; ${ }^{7}$ Ibaraki Higashi Hospital; ${ }^{8}$ Mito Kyodo General Hospital, Ibaraki, Japan
}

Received July 21, 2011; Accepted September 9, 2011

DOI: 10.3892/etm.2011.360

\begin{abstract}
There is no standard treatment and there are no clearly defined guidelines for the treatment of postoperative recurrent non-small-cell lung cancer (NSCLC). We performed a retrospective population-based study to assess the benefits of treatment with gefitinib in patients with a postoperative recurrence of NSCLC in general clinical practice. This retrospective population-based study was conducted on patients with postoperative recurrent NSCLC who had been treated with gefitinib at 14 institutions in Ibaraki Prefecture between July 2002 and September 2007. The objective response rate to gefitinib therapy was $37.6 \%$ for local and distant recurrence. The median survival time following the start of gefitinib therapy was 12 months, and the one-year and two-year survival rates were 48.9 and $28.9 \%$, respectively. The median survival time of the females was 19 months, and the median survival time of the males was 9 months ( $\mathrm{p}=0.002)$. Univariate analysis showed that female gender, adenocarcinoma, a performance status (PS) of 0-1 and absence of smoking history were favorable prognostic factors. Only female gender and a PS of 0-1 were independent statistically significant prognostic factors in the multivariate analysis. The rate of greater than grade 1 interstitial lung damage as an adverse event was 3.5\%. Gefitinib is a feasible treatment for postoperative recurrent NSCLC in general clinical practice, and a good response and prolonged survival were obtained, similar to the findings reported in published clinical studies that were conducted on highly selected patients.
\end{abstract}

Correspondence to: Dr Kinya Furukawa, Department of Chest Surgery, Tokyo Medical University, Ibaraki Medical Center, 3-20-1 Chuo, Ami-machi, Inashiki-gun, Ibaraki 300-0395, Japan E-mail: k-furu@tokyo-med.ac.jp

Key words: gefitinib, Iressa, recurrence, lung cancer, surgery, population-based study

\section{Introduction}

Surgical resection is considered to be the most effective treatment for stage I-IIIA non-small-cell lung cancer (NSCLC). Despite curative surgery, however, numerous patients are diagnosed with distant or local recurrence following surgery, and succumb to the disease as a result of the recurrent lung cancer. Since there is no standard treatment and there are no clearly defined guidelines for the treatment of patients with postoperative recurrence, chemotherapy and/or radiotherapy are usually considered, depending on the patient's condition, the same as in cases of inoperable advanced NSCLC.

The orally active epidermal growth factor receptor (EGFR) tyrosine kinase inhibitor gefitinib (Iressa, AstraZeneca, Wilmington, DE, USA) has been demonstrated to have significant antitumor efficacy and provide significant symptom relief in patients with NSCLC previously treated by chemotherapy that included cis-diamminedichtoroplatinum (CDDP) in a Phase II study (IDEAL-1, 2) $(1,2)$. Based on the results of the IDEAL studies, in 2002, gefitinib was approved in Japan for the treatment of inoperable and recurrent NSCLC. Recent clinical phase studies of patients with advanced and recurrent NSCLC have shown a correlation between gefitinib response and a number of clinical factors, including adenocarcinoma, absence of smoking history, female gender and Asian ethnicity. (3-7)

Gefitinib treatment has already become a treatment option in cases of recurrent NSCLC following surgical resection as well as in inoperable advanced cases of NSCLC in general clinical practice in Japan. We believe that gefitinib treatment should also be evaluated by means of population-based studies, since the subjects of clinical trials tend to consist of highly selected populations that are not representative of NSCLC patients in general clinical practice. We previously reported the first population-based study of advanced and recurrent NSCLC treated with gefitinib in general clinical practice in Japan (8). The results obtained, particularly with respect to response rate, median survival time (MST), 1-year survival, and adverse effects, were comparable to those reported in previously published clinical trials whose criteria restricted eligibility.

Since reports on the benefits of gefitinib specifically in patients with postoperative recurrent NSCLC have been rare, 
and the impact of gefitinib on population-based outcomes is difficult to measure, in the present study, we performed a subgroup analysis of NSCLC patients with recurrence following surgery who were selected from the subjects in our previous report, who consisted of patients with inoperable advanced NSCLC and patients with postoperative recurrent NSCLC. To assess the benefits of gefitinib in patients with postoperative recurrent NSCLC, in this population-based study we retrospectively reviewed the characteristics, response to therapy, toxicity and survival of patients with recurrent NSCLC treated with gefitinib.

\section{Patients and methods}

Ibaraki Prefecture, Japan, has an area of $6095 \mathrm{~km}^{2}$ and a population of 3 million. This retrospective population-based study was conducted by reviewing the records of patients with NSCLC whose treatment had included gefitinib at 14 institutions (18 divisions) in Ibaraki Prefecture between July 2002 and September 2007. A pathological or cytological diagnosis of NSCLC was required for inclusion in this study. The pathological diagnosis of lung cancer was made according to the WHO classification. Recurrence following surgery was diagnosed by chest computed tomography (CT), brain magnetic resonance imaging (MRI), bone scintigraphy, and ultrasonography and/ or CT of the abdomen during follow-up examinations. Eligible cases were identified in the clinical database of each hospital, and the following information was extracted from the data: data at the time of the gefitinib therapy (age, gender, smoking history, histological diagnosis and pathological stage), and objective tumor response. Tumor response was evaluated according to the Response Evaluation Criteria in Solid Tumors as complete response (CR), partial response (PR), stable disease (SD), progressive disease (PD), or not evaluable (NE). Toxicity was investigated with regard to interstitial lung damage (ILD). Overall survival time was defined as the interval between the start of gefitinib treatment and the date of mortality or the last follow-up examination. This study plan was approved by each institution's Institutional Review Board.

EGFR mutation data were provided by two institutions. Surgical specimens were examined for mutations by direct sequencing of clones. Samples were digested with SDS and proteinase $\mathrm{K}$, and DNA was extracted by using a DNA extractor WB kit (Wako Pure Chemical Industries, Ltd., Osaka, Japan) according to the manufacturer's instructions. Exons 18, 19 and 21 of the EGFR gene were amplified by using the following primers: exon 18-forward, 5'-CTG TGT TCT TGT CCC CCC CAG-3' and reverse, 5'-GGC CTG TGC CAG GGA CCT TAC-3'; exon 19-forward, 5'-TTC CTT CTC TCT CTG TCA TAG-3' and reverse, 5'-CAC AGC AAA GCA GAA ACT CAC-3'; and exon 21-forward, 5'-GCA GGG TCT TCT CTG TTT CAG-3' and reverse, 5'-GAC CTA AAG CCA CCT CCT TAC-3', and the sizes of the PCR products were $165 \mathrm{bp}$ (exon 18), 141 bp (exon 19), and $198 \mathrm{bp}$ (exon 21). We also evaluated the correlation between the presence of a mutation and the antitumor efficacy of gefitinib therapy.

Statistical analysis. Differences in proportions between two independent subgroups were compared by the Chi-square test.
Table I. Patient profiles.

Total no. of patients

Recurrent site

Local

Distant

Follow-up (months)

Median

Range

Age (years)

Median

Range

Histology

Adenocarcinoma

Non-adenocarcinoma

Gender

Female

Male

Smoking status

Non-smoker

Smoker

Unknown

PS

0-1

96

2-4

Gefinitib Tx line

First

Second

Third

Tumor response

CR, PR

SD

PD

NE

A value of $\mathrm{p}<0.05$ was considered to be statistically significant. In the analysis of survival, time zero was defined as the date gefitinib administration was initiated. Survival probability was estimated by the Kaplan-Meier method and compared using the log-rank test. Multivariate analysis of significant prognostic factors identified by the univariate analysis was performed by using Cox's proportional hazard model.

\section{Results}

Patient characteristics. Complete data sets of the 141 patients with recurrence following surgery were obtained from the records of a total of 626 NSCLC patients who had received gefitinib. The other 485 patients had advanced inoperable NSCLC. The characteristics of the patients with postoperative recurrence are shown in Table I. There were 48 patients with local recurrence $(34 \%)$ and 93 patients with distant metastasis $(66 \%)$. The median follow-up period after the 
Table II. Benefits and risks of each group of patients.

\begin{tabular}{|c|c|c|c|c|c|}
\hline & \multirow[b]{2}{*}{$\mathrm{n}$} & \multicolumn{3}{|c|}{ Benefit } & \multirow{2}{*}{$\frac{\text { Risk }}{\operatorname{ILD}(\%)^{\mathrm{a}}}$} \\
\hline & & $\mathrm{CR} / \mathrm{PR}(\%)$ & $\operatorname{MST}(\mathrm{m})$ & $95 \% \mathrm{CI}$ & \\
\hline Overall & 141 & $53(37.6)$ & 12 & $6.4-17.6$ & $5(3.5 \%)$ \\
\hline \multicolumn{6}{|c|}{ Adeno, non-smoker } \\
\hline Female & 55 & $24(43.6)$ & 22 & $15.8-28.2$ & $1(1.8 \%)$ \\
\hline Male & 10 & $4(40.0)$ & 15 & $11.0-19.0$ & $0(0.0 \%)$ \\
\hline \multicolumn{6}{|l|}{ Adeno, smoker } \\
\hline Female & 16 & $7(43.8)$ & 24 & 14.4-33.6 & $1(6.3 \%)$ \\
\hline Male & 38 & $12(31.6)$ & 7 & $4.8-9.3$ & $2(5.3 \%)$ \\
\hline \multicolumn{6}{|l|}{ Non-adeno } \\
\hline Non-smoker & 4 & $1(25.0)$ & 4 & - & $0(0 \%)$ \\
\hline Smoker & 16 & $4(25.0)$ & 6 & $2.9-9.1$ & $1(6.3 \%)$ \\
\hline
\end{tabular}

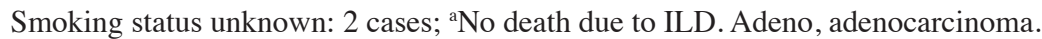

\section{Overall survival after initiation of Gefitinib}

A

Post-operative recurrent cases $(n=141)$

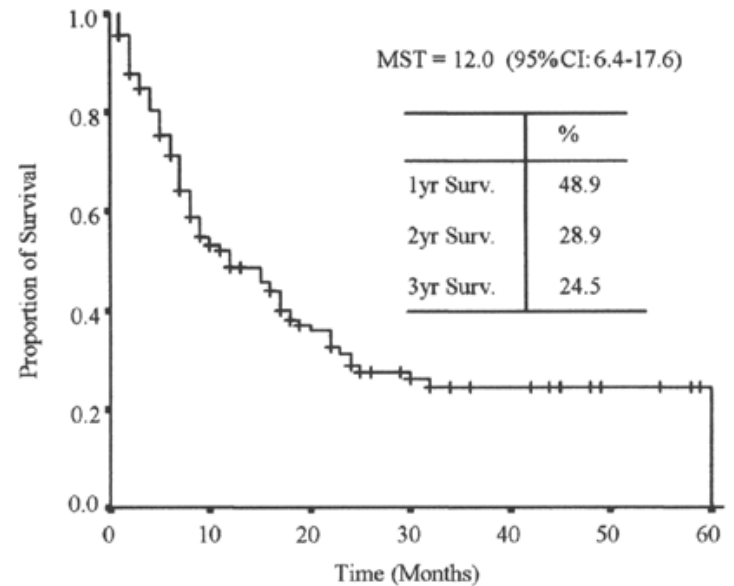

B

Advanced inoperative cases $(n=485)$

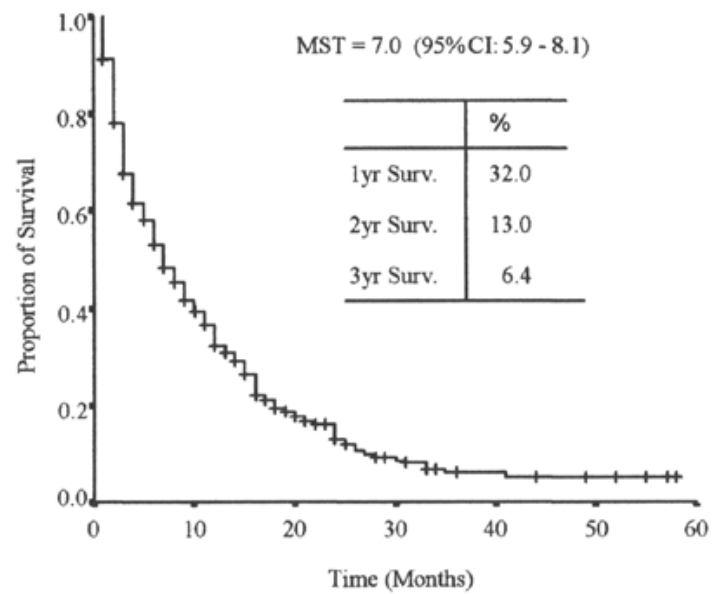

Figure 1. The MST of the patients with postoperative recurrence was 12.0 months (95\% CI; 6.4-17.6 months), and their 1-year and 2-year survival rates were $48.9 \%$ and $28.9 \%$, respectively. The MST of patients with advanced inoperable disease was 7.0 months (95\% CI; 5.9-8.1 months), and their 1-year and 2-year survival rates were 32.0 and $13.0 \%$, respectively.

initiation of gefitinib therapy was 9 months (1-60 months). The median age was 65 years (range 33-86), and $54.6 \%$ of the patients were female. The histological diagnosis in $85.8 \%$ of the patients was adenocarcinoma, $48.9 \%$ of the patients were non-smokers, and $68.1 \%$ of the patients had an ECOG performance status (PS) of 0-1.

Response to treatment. The objective response rate (ORR) to gefitinib therapy was $37.6 \%$ (53 $\mathrm{CR}+\mathrm{PR})$. The response was evaluated as SD in $25.5 \%$ of the patients, and the disease control rate was $63.1 \%$. A total of 27 of the 141 patients were not evaluated (NE) for tumor response. Higher responses were documented in the females with adenocarcinoma than in the males with adenocarcinoma among non-smokers and smokers (non-smokers, 43.6 vs. $40 \%$; smokers, 43.8 vs. $31.6 \%$; Table II).
Survival analysis. The MST was 12 months [95\% confidence interval (CI), 6.4-17.6 months], and the 1-year and 2-year survival rates were $48.9 \%$ and $28.9 \%$, respectively (Fig. 1A). The MST of the patients with advanced inoperable NSCLC was 7 months (95\% CI, 5.9-8.1 months), and their 1-year and 2 -year survival rates were $32 \%$ and $13 \%$, respectively (Fig. 1B). Survival after the initiation of gefitinib therapy was improved in the group with postoperative recurrence as opposed to the group with advanced inoperable NSCLC. The subgroup analysis of postoperative recurrence according to histological diagnosis, gender and smoking history (Table II) showed a higher MST in the female subgroup with adenocarcinoma among the non-smokers (22 months; 95\% CI, 15.8-28.2) and smokers (24 months; 95\% CI, 14.3-33.6) than in the male subgroup with adenocarcinoma among the non-smokers 
Table III. Univariate analysis of clinical features.

\begin{tabular}{|c|c|c|c|c|}
\hline Variable & $\mathrm{N}$ & MST (months) & $95 \% \mathrm{CI}$ & P-value \\
\hline Gender & & & & 0.0022 \\
\hline Female & 77 & 19 & $13.8-24.3$ & \\
\hline Male & 64 & 9 & $7.2-10.8$ & \\
\hline Histology & & & & 0.0304 \\
\hline Adenocarcinoma & 121 & 16 & $12.0-20.0$ & \\
\hline Non-adenocarcinoma & 20 & 6 & $2.7-9.3$ & \\
\hline Female & & & & 0.0694 \\
\hline Adenocarcinoma & 73 & 22 & $16.1-27.9$ & \\
\hline Non-adenocarcinoma & 4 & 2 & $0.0-4.9$ & \\
\hline Male & & & & 0.5602 \\
\hline Adenocarcinoma & 48 & 10 & $6.2-13.8$ & \\
\hline Non-adenocarcinoma & 16 & 8 & $5.7-10.3$ & \\
\hline Smoking status & & & & 0.0243 \\
\hline Smoker & 70 & 8 & $6.3-9.7$ & \\
\hline Non-smoker & 69 & 17 & $10.8-23.2$ & \\
\hline Female & & & & 0.7518 \\
\hline Smoker & 17 & 19 & $9.2-28.8$ & \\
\hline Non-smoker & 58 & 22 & $15.6-28.4$ & \\
\hline Male & & & & 0.1544 \\
\hline Smoker & 53 & 8 & $6.3-9.7$ & \\
\hline Non-smoker & 11 & 15 & $11.0-19.0$ & \\
\hline PS & & & & 0.0006 \\
\hline $0-1$ & 96 & 17 & $9.6-24.4$ & \\
\hline $2-4$ & 45 & 8 & $5.6-10.6$ & \\
\hline Female & & & & 0.0001 \\
\hline $0-1$ & 55 & 32 & $20.7-43.3$ & \\
\hline $2-4$ & 22 & 7 & $2.6-11.5$ & \\
\hline Male & & & & 0.5012 \\
\hline $0-1$ & 41 & 8 & $4.3-11.3$ & \\
\hline $2-4$ & 23 & 9 & $6.7-11.3$ & \\
\hline Gefitinib Tx line & & & & 0.8907 \\
\hline First & 25 & 10 & $0.0-26.3$ & \\
\hline Second & 57 & 16 & $12.6-19.4$ & \\
\hline Third & 59 & 9 & $6.0-12.0$ & \\
\hline Tumor response & & & & $<0.0001$ \\
\hline $\mathrm{CR} / \mathrm{PR}$ & 53 & 24 & $13.5-34.5$ & \\
\hline SD & 36 & 17 & $8.3-25.7$ & \\
\hline PD & 25 & 4 & $1.6-6.5$ & \\
\hline
\end{tabular}

(15 months; 95\% CI, 11.0-19.0) and smokers (7 months; 95\% CI, 4.8-9.3).

To identify factors that affected survival, a univariate analysis was performed with gender, PS, smoking history and histological diagnosis as covariates (Table III). The MST of the females was 19 months (95\% CI; 13.8-24.3), the MST of the males was 9 months (95\% CI; 7.2-10.8) and the difference was statistically significant ( $\mathrm{p}=0.0022)$. As shown in Table III, adenocarcinoma, a PS of 0-1 and absence of smoking history, as well as female gender, were favorable prognostic factors in the univariate analysis. The MST of the patients who received gefitinib as first-line therapy, second-line therapy and third- or later line therapy was 10 (95\% CI, 0-26.3 months), 16 (95\% CI, 12.6-19.4 months), and 9 months (95\% CI, 6.0-12.0 months), respectively, and there were no statistically significant differences in survival time according to the therapy line ( $\mathrm{p}=0.8907$ ). A multivariate analysis of the significant prognostic factors identified in the univariate analysis was carried out using Cox's proportional hazard model, and the results showed that female gender [hazard ratio (HR), 0.575; 95\% CI, 0.374-0.886; 
Table IV. COX analysis proportional hazards model of survival.

\begin{tabular}{lccc}
\hline Variable & HR & $95 \%$ CI & P-value \\
\hline Female/male & 0.575 & $0.374-0.886$ & 0.012 \\
PS 0,1/2-4 & 0.543 & $0.351-0.840$ & 0.006 \\
\hline
\end{tabular}

Excluded variables: smoking and histology; Stepwise methods (include $<0.05$, exclude $>0.2$ ).

Table V. EGFR mutation and clinical features $(n=35)$.

\begin{tabular}{|c|c|c|c|c|c|}
\hline \multirow[b]{2}{*}{ Variable } & \multicolumn{2}{|c|}{ Mutant } & \multicolumn{2}{|c|}{ Wild-type } & \multirow[b]{2}{*}{ P-value } \\
\hline & $\mathrm{n}$ & $\%$ & $\mathrm{n}$ & $\%$ & \\
\hline Gender & & & & & 0.0055 \\
\hline Female & 9 & 60.0 & 6 & 40.0 & \\
\hline Male & 3 & 15.0 & 17 & 85.0 & \\
\hline Smoking status & & & & & 0.0127 \\
\hline Smoker & 2 & 12.5 & 14 & 87.5 & \\
\hline Non-smoker & 10 & 52.6 & 9 & 47.4 & \\
\hline Histology & & & & & 0.5287 \\
\hline Adenocarcinoma & 10 & 37.0 & 17 & 63.0 & \\
\hline Non-adenocarcinoma & 2 & 25.0 & 6 & 75.0 & \\
\hline Response & & & & & 0.1572 \\
\hline $\mathrm{CR} / \mathrm{PR}$ & 6 & 50.0 & 6 & 26.1 & \\
\hline $\mathrm{SD} / \mathrm{PD}$ & 6 & 50.0 & 17 & 73.9 & \\
\hline Total & 12 & 34.3 & 23 & 65.7 & \\
\hline
\end{tabular}

$\mathrm{p}<0.012]$ and a PS of 0-1 (HR, 0.543; 95\% CI, 0.351-0.840; $\mathrm{p}<0.006)$ were independent statistically significant prognostic factors (Table IV).

Toxicity. The risks of ILD are shown in Table II. ILD adverse events greater than grade 1 were reported in $5(3.5 \%)$ of the 141 patients, but there was no mortality due to ILD (no cases of grade 5 toxicity). The subgroup analysis according to adenocarcinoma histology, gender and smoking history showed a lower incidence of ILD in the non-smoking group (0-1.8\%) than in the smoking group (5.3-6.3\%).

EGFR mutation and clinical features. EGFR mutation data for 35 patients were available from only 2 institutions, as the cost of testing for EGFR mutations was not reimbursed by health insurance at the time of this study. As shown in Table V, a mutation was detected in $60 \%$ of the females, $52.6 \%$ of those with no smoking history and $37 \%$ of those with adenocarcinoma (34.3\% of the patients overall). There were significant differences in the EGFR mutation rate according to gender $(\mathrm{p}=0.0055)$ and smoking status $(\mathrm{p}=0.0127)$ based on the results of the Chi-square test. The tumor response rate in the mutation-positive group was higher than in the mutation-negative group, but the difference was not signifi-

\section{Overall survival by EGFR mutation}

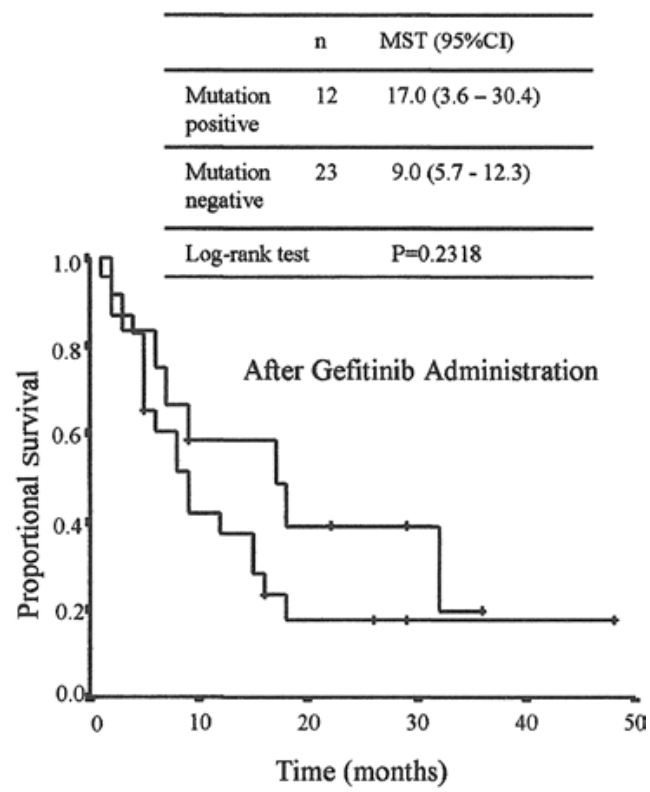

Figure 2. The MST of patients with postoperative recurrence of NSCLC was 17 months (95\% CI; 3.8-30.4 months) in the EGFR-mutation-positive, group and 9 months (95\% CI; 5.7-12.3 months) in the wild-type group, but the difference between the groups was not statistically significant $(p=0.2318)$ due to the small number of patients.

cant $(50 \%$ vs. $26.1 \%, \mathrm{p}<0.1572)$. As shown in Fig. 2, overall survival following the initiation of gefitinb therapy differed according to mutation status. MST was 17 months $(95 \% \mathrm{CI}$; 3.6-30.4 months) in the mutation-positive group and 9 months (95\% CI; 5.7-12.3 months) in the wild-type group, but due to the small number of patients, the difference was not statistically significant $(\mathrm{p}=0.2318)$.

\section{Discussion}

Molecularly targeted therapy with gefitinib has already become a treatment option similar to chemotherapy in cases of recurrence following surgical resection as well as in inoperable advanced cases in general clinical practice in Japan. However, since the efficacy of chemotherapeutic agents or gefitinib in cases of recurrence following surgery and advanced inoperable cases may differ, we evaluated the effectiveness of gefitinib in recurrent NSCLC following surgery. The survival time and survival rate after the initiation of gefitinib therapy in the group with recurrence following surgery in this population-based study were improved as opposed to the group with advanced inoperable NSCLC (MST, 12 vs. 7 months; two-year survival, 28.9 vs. 13\%). In 2001, prior to the approval of gefitinib in Japan, Yoshino et al reviewed the cases of a total of 118 patients with recurrence following surgery for NSCLC and showed that systemic chemotherapy tended to prolong survival (MST, 12 months; two-year survival, $19 \%$ after recurrence), but the difference between the group that received chemotherapy and the control group was not statistically significant ( $\mathrm{p}=0.0928)(9)$. Our results for survival following the initiation of gefitinib therapy, but not after the diagnosis of recurrence, showed improved survival (MST, 12 months; two-year survival, 28.9\%) compared to the study 
by Yoshino et al. The data obtained in our study suggest that treatment including gefitinib is superior to chemotherapy alone without gefitinib in NSCLC patients with recurrence following surgery. Therefore, we should try to treat patients with recurrence following surgery with gefitinib.

As the effectiveness of gefitinib against adenocarcinoma was particularly well known at the time it was released on the market based on the data from the IDEAL-1 and -2 studies $(1,2)$, general practitioners were already prescribing gefitinib for $86 \%$ of the patients with adenocarcinoma in this study. Initially, general practitioners may attach importance to adenocarcinoma histology rather than gender or smoking habit, since the numbers of males or females, and of smokers or non-smokers in this study were almost the same. Since a number of recent clinical studies of gefitinib in inoperable NSCLC have shown a correlation between gefitinib response and a number of clinical factors, including histological diagnosis of adenocarcinoma, no history of smoking, female gender, Asian ethnicity and EGFR-mutation-positive status (3-7), general practitioners have started to consider some of these factors, such as female gender and EGFR-mutation-positive status. Mitsudomi et al demonstrated that patients with recurrence following resection who were female, non-smokers and had adenocarcinoma tended to respond better to gefitinib therapy (7). The subgroup analysis in our own study showed a longer MST in the adenocarcinoma, female gender and non-smoker subgroups. Interestingly, the female patients had a favorable MST of more than 20 months regardless of smoking status, while the male smokers had a poor MST of only 7 months. Gefitinib should be considered a treatment option for women even when they are smokers. Women with a PS of 0-1 had a longer MST than women whose PS was 2-3. By contrast, male patients with a PS of 0-1 did not have a longer MST than males whose PS was 2-3. PS does not seem to be a significant clinical prognostic factor in male patients. However, while smoking history, gender and PS were highly confounded with each other, gefitinib is expected to be effective in female non-smokers with a PS of $0-1$, the same as in previously reported clinical trials.

A cohort and nested case-control study concluded that ILD was relatively common in Japanese patients with NSCLC who were being treated with gefitinib, and that its incidence was higher in older, smoking patients with preexisting interstitial pneumonitis or poor PS (10). The incidence of ILD in our own study was $3.5 \%$ overall, and almost the same as in previous reports $(10,11)$. The incidence was higher in smokers regardless of the histological type of their NSCLC.

Although the addition of gefitinib to standard first-line chemotherapy does not provide a clinical benefit over chemotherapy alone in patients with advanced or metastatic NSCLC $(12,13)$, recent studies have demonstrated the usefulness of first-line monotherapy with gefitinib in advanced NSCLC. The results of the Iressa Pan-Asia Study (IPASS) showed superior progression-free survival (PFS) in the gefitinib group than in a group treated with a combination of carboplatin and paclitaxel as first-line treatment of East Asian patients who were non- or light-smokers and had a histological diagnosis of adenocarcinoma (14). A subgroup analysis in that study showed that the presence of a mutation of the EGFR gene in the tumor is a strong predictor of an improved outcome with gefitinib (HR for progression or death, 0.48; 95\% CI,
0.36-0.64; $\mathrm{P}<0.001)$. Also, in a Japanese study of Iressa, the combined survival analysis of the mutation positive (I-CAMP) study group identified seven eligible trials for NSCLC patients with EGFR mutations who were treated with first-line gefitinib (15). The median PFS after the start of first-line therapy was significantly longer in the gefitinib-first group than in the chemotherapy-first group (10.7 vs. 6 months; $p<0.001)$ in the I-CAMP study. The International Expert Panel Meeting on the first-line treatment of advanced NSCLC organized by the Italian Association of Thoracic Oncology recommended that every effort be made to obtain adequate tumor tissue prior to initiation of treatment (16).

An EGFR mutation analysis should be performed in subgroups of patients characterized by a higher prevalence of sensitizing mutations (Asians, never-smokers, females and patients with adenocarcinoma), and first-line treatment with a single-agent EGFR tyrosine-kinase inhibitor may be considered when a mutation is present. In our own retrospective study, there were no significant differences in survival between the first-line, second-line, and third- or later-line groups. Although first-line treatment with gefitinib is considered for patients with poor PS who cannot tolerate systemic chemotherapy, since gefitinib was administered to $64 \%$ of the patients with a PS of 0-1, clinical factors other than poor PS, such as the presence of complications or patient refusal of systemic chemotherapy, may affect the selection of first-line gefitinib treatment in general clinical practice.

A number of studies on recurrent NSCLC following surgery that was treated with gefitinib reported ORRs of $33-56.5 \%$, EGFR mutation rates of 44.1-58.8\%, and response rates in EGFR-mutation-positive groups of $65-92 \%$. MST in the mutation-positive cases was 16-31 months, and the significant prognostic factors according to the results of a multivariate analysis were EGFR mutation and disease-free interval $(7,17,18)$. Our data regarding the percentage of mutations $(34.3 \%)$ and response rate of mutations (50\%) were slightly lower than those data. The differences may be explained by the fact that there were fewer EGFR mutation tests in our study, as their cost was not reimbursed by health insurance at the time of this study. The other data in our study (ORR, 37.6\%; MST in mutations, 17 months) were similar to the data obtained in other studies. Significant prognostic factors identified in the multivariate analysis were female gender and a PS of 0-1. However, we did not include EGFR mutation in the analysis as so few patients were tested. The Nagoya University Group analyzed primary tumor specimens obtained from lung cancer patients at initial surgery for the presence of EGFR mutations, and investigated whether the response of recurrent tumors to gefitinib depended on the presence of the activating mutation (19). The results of their study suggested that analyzing specimens obtained at surgery for EGFR mutations may be useful in selecting the appropriate treatment for patients with recurrent lung cancer.

In the present study, gefitinib therapy for the recurrence of NSCLC following surgical resection was found to be superior in terms of survival to gefitinib therapy for inoperable advanced NSCLC in general clinical practice, and gefitinib therapy should be considered for patients with postoperative recurrent NSCLC who are female, who have adenocarcinoma, a PS of $0-1$, are non-smokers, and whose cancer is EGFR mutation- 
positive, similar to published clinical phase studies conducted on highly selected patients. Gefitinib is a feasible treatment for patients with recurrence of NSCLC following surgery, and a good response and longer survival may be obtained. Since treatments that included gefitinib were demonstrated to be more useful than cytotoxic chemotherapy regimens alone in patients with postoperative recurrent NSCLC in this study, gefitinib should be considered for the treatment of recurrent NSCLC following surgery. Further prospective investigation of EGFR mutations may support the findings in this study.

\section{Acknowledgements}

The authors of this study all belong to the Ibaraki Advanced Lung Cancer Study Group. The authors are indebted to $\mathrm{Mr}$ Roderick J. Turner and Associate Professor Raoul Breugelmans of the Department of International Medical Communications of Tokyo Medical University for their review of this manuscript.

\section{References}

1. Fukuoka M, Yano S, Giaccone G, et al: Multi-institutional randomized phase II trial of gefitinib for previously treated patients with advanced non-small-cell lung cancer (The IDEAL 1 Trial). J Clin Oncol 21: 2237-2246, 2003.

2. Kris MG, Natale RB, Herbst RS, et al: Efficacy of gefitinib, an inhibitor of the epidermal growth factor receptor tyrosine kinase, in symptomatic patients with non-small cell lung cancer: a randomized trial. JAMA 290: 2149-2158, 2003.

3. Ham SW, Kim TY, Lee KH, et al: Clinical predictors versus epidermal growth factor receptor mutation in gefitinib-treat non-small-cell lung cancer patients. Lung Cancer 54: 201-207, 2006.

4. Chang GC, Tsai CM, Chen KC, et al: Predictive factors of gefitinib antitumor activity in East Asian advanced non-small cell lung cancer patients. J Thorac Oncol 1: 520-525, 2006.

5. Itaya T, Yamaoto N, Ando M, et al: Influence of histological type, smoking history and chemotherapy on survival after first-line therapy in patients with advanced non-small cell lung cancer. Cancer Sci 98: 226-230, 2007.

6. Lynch TJ, Bell DW, Sordella R, et al: Activating mutations in the epidermal growth factor receptor underlying responsiveness of non-small-cell lung cancer to gefitinib. N Eng J Med 350: 2129 2139,2004

7. Mitsudomi T, Kosaka T, Endoh H, et al: Mutations of the epidermal growth factor receptor gene predict prolonged survival after gefitinib treatment in patients with non-small-cell lung cancer with postoperative recurrence. J Clin Oncol 23: 2513-20, 2005.

8. Hayashibara K, Satoh H, Shinohara Y, et al: A population-based study of gefitinib in patients with non-small cell lung cancer. Med Oncol 26: 222-227, 2009.

9. Yoshino I, Yohena T, Kitajima M, Ushijima C, Nishioka K, Ichinose Y and Sugimachi K: Survival of non-small cell lung cancer patients with postoperative recurrence at distant organs. Ann Thorac Cardiovasc Surg 7: 204-209. 2001.
10. Kudoh S, Kato H, Nishiwaki Y, et al: Interstitial lung disease in Japanese patients with lung cancer: a cohort and nested case-control study. Am J Respir Crit Care Med 177: 1348-1357, 2008.

11. Nakagawa M, Nishimura T, Teramukai S, et al: Interstitial lung disease in gefitinib-treated Japanese patients with non-small cell lung cancer - a retrospective analysis: JMTO LC03-02. BMC Res Notes 2: 157, 2009.

12. Giaccone G, Herbst RS, Manegold C, et al: Gefitinib in combination with gemcitabine and cisplatin in advanced non-small-cell lung cancer: a phase III trial--INTACT 1. J Clin Oncol 22: 777-784, 2004.

13. Herbst RS, Giaccone G, Schiller JH, et al: Gefitinib in combination with paclitaxel and carboplatin in advanced non-small-cell lung cancer: a phase III trial-INTACT 2. J Clin Oncol 22: 785-794, 2004

14. Mok TS, Wu YL, Thongprasert S, et al: Gefitinib or carboplatin-paclitaxel in pulmonary adenocarcinoma. N Engl J Med 361: 947-957, 2009

15. Morita S, Okamoto I, Kobayashi K, et al: Combined survival analysis of prospective clinical trials of gefitinib for non-small cell lung cancer with EGFR mutations. Clin Cancer Res 15: 4493-4498, 2009.

16. Gridelli C, Ardizzoni A, Douillard JY, et al: Recent issues in first-line treatment of advanced non-small-cell lung cancer: Results of an International Expert Panel Meeting of the Italian Association of Thoracic Oncology. Lung Cancer 68: 319-331, 2010.

17. Shoji F, Yano T, Yoshino I, Mori D, Yamasaki F, Kohno H and Maehara Y: The characteristics and failure pattern of gefitinib responders with postoperative recurrence of pulmonary adenocarcinoma. Eur J Surg Oncol 34: 89-93, 2008.

18. Okami J, Taniguchi K, Higashiyama M, et al: Prognostic factors for gefitinib-treated postoperative recurrence in non-small cell lung cancer. Oncology 72: 234-242, 2007.

19. Kondo M, Yokoyama T, Fukui T, et al: Mutations of epidermal growth factor receptor of non-small cell lung cancer were associated with sensitivity to gefitinib in recurrence after surgery. Lung Cancer 50: 385-391, 2005.

\section{Appendix}

The following principal investigators and institutions also belonged to the Ibaraki Advanced Lung Cancer Study Group and participated in this study: Saito T, Ibaraki Higashi Hospital; Kurishima K, Ohtsuka M, Hizawa N, Gotoh Y, Onizuka M, and Satoh Y, University of Tsukuba Hospital; Shinohara Y, Tsuchiura Kyodo General Hospital; Ishikawa H, Tsukuba Medical Center Hospital; Yamamoto Y, Hitachi General Hospital; Nawa T, Hitachi General Hospital; Funayama Y, Tsukuba Gakuen General Hospital; Matsumura T, Ibaraki Seinan General Hospital; Kagohashi K; Mito Kyodo General Hospital; Endo T, Mito Medical Center Hospital; Sumi M, Kensei General Hospital; Kashimoto T, Mito Saiseikai Hospital. 